Dear author,

Please note that changes made in the online proofing system will be added to the article before publication but are not reflected in this PDF.

We also ask that this file not be used for submitting corrections. 


\title{
Diffraction of antiplane shear waves and stress concentration in a cracked couple stress elastic material with micro inertia
}

\author{
Andrea Nobili ${ }^{a}, *$ Enrico Radi ${ }^{b}$, Adam Vellender $^{c}$ \\ a Dipartimento di Ingegneria Enzo Ferrari, via Vivarelli 10, Modena, Italy \\ ${ }^{\mathrm{b}}$ Dipartimento di Scienze e Metodi dell'Ingegneria, via Amendola 2, Reggio Emilia, 42122, Italy \\ ${ }^{\mathrm{c}}$ Institute of Mathematical and Physical Sciences, Aberystwyth University, Ceredigion, Wales, SY23 3BZ, UK
}

\section{A R T I C L E I N F O}

\section{Article history:}

Received 28 August 2018

Revised 8 November 2018

Accepted 23 November 2018

Available online $\mathrm{xxx}$

\section{Keywords:}

Couple stress

Wave diffraction

Rayleigh waves

Dynamic stress intensity factor

\begin{abstract}
A B S T R A C T
We investigate diffraction of reduced traction shear waves applied at the faces of a stationary crack in an elastic solid with microstructure, under antiplane deformation. The material behaviour is described by the indeterminate theory of couple stress elasticity and the crack is rectilinear and semi-infinite. The full-field solution of the crack problem is obtained through integral transforms and the Wiener-Hopf technique. A remarkable wave pattern appears which consists of entrained waves extending away from the crack, reflected Rayleigh waves moving along the crack, localized waves irradiating from the cracktip with, possibly, super-Rayleigh speed and body waves scattered around the crack-tip. Interestingly, the localized wave solution may be greatly advantageous for defect detection through acoustic emission. Dynamic stress intensity factors are presented, which generalize to Elastodynamics the corresponding results already obtained in the static framework. The correction brings out the important role of wave diffraction on stress concentration.
\end{abstract}

(c) 2018 Published by Elsevier Ltd.

\section{Introduction}

The study of wave diffraction has attracted major interest since its discovery, in the XVII century, by Francesco Maria Grimaldi in the context of light wave propagation (see Mow and Pao, 1971 for an excellent historical account). Indeed the term diffraction was then introduced to indicate a deviation from the rectilinear path which could not be accounted for by either reflection or refraction. Starting from the pioneering work by Clebsch (1863) and Strutt (1877), diffraction of elastic waves by inclusions, barriers and obstacles has been investigated in a vast body of literature. Nonetheless, only in fairly recent times could the importance of dynamic effects in determining the stress concentration in the presence of geometric discontinuities be appreciated. Indeed, "dynamic stress concentration is a result of diffraction of elastic wave" (Mow and Pao, 1971).

Elastic wave diffraction and stress concentration are almost always investigated within the classical theory of Elastodynamics, which fails to account for the discontinuous nature of many engineering materials, the so-called microstructure. As an example, this theory cannot predict dispersion of Rayleigh waves at high frequency, when the wavelength becomes comparable to the material characteristic length (Georgiadis and Velgaki, 2003). Besides, the discrepancy between the classical theory and the experimental evidence is more pronounced for those complex materials, such as composites, cellular

\footnotetext{
* Corresponding author.

E-mail address: andrea.nobilii@unimore.it (A. Nobili).
} 
materials, foams, masonry, bone tissues, glassy and semicrystalline polymers, for which modelling is most needed. In this respect, wave diffraction and microstructural effects are deeply related to each other and together contribute to the determination of the stress concentration near geometric discontinuities.

The use of enhanced constitutive models based on strain gradient or nonlocal theories of elasticity allows us to circumvent some of the pathological results provided by the classical theory of elasticity (Georgiadis and Vardoulakis, 1998). However, the number of material parameters is comparatively large (Lam et al., 2003; Mindlin, 1964; Mindlin and Eshel, 1968), so that a substantial effort is required on the experimental side for their determination (Maranganti and Sharma, 2007). Furthermore, analytical solutions are most often inaccessible (Gao and Ma, 2010).

An intermediate step between the classical elastic theory and the most advanced strain gradient theories is provided by the Cosserat micropolar model, which involves only rotational gradients (Graff and Pao, 1967). A special class of micropolar theories is represented by the indeterminate couple stress (CS) theory, developed by Koiter (1964) for the quasi-static regime and later extended by Eringen (1999) to Elastodynamics. Alongside the traditional Lamé moduli, this elastic constitutive model features two extra material characteristic lengths, associated to bending and torsion, as well as the micropolar rotatory inertia.

Comparably few contributions can be found in the literature discussing wave propagation in solids with microstructure. The original contribution by Graff and Pao (1967) considers wave reflection by a rigid obstacle in a CS half-space under plane strain. More recently, a similar treatment is given in Gourgiotis et al. (2013) for a grade two strain-gradient material featuring three material constants. Rayleigh waves propagating in CS materials are investigated in Ottosen et al. (2000), in the absence of rotational inertia, and then in Georgiadis and Velgaki (2003) accounting for rotational inertia, again under plane strain. Scattering of antiplane shear waves caused by a cylindrical inclusion within the CS theory is considered by Shodja et al. (2015).

Enhanced models of continua may result in new types of surface waves, for example the appearance of new surface antiplane waves in a half-space with surface stresses (Eremeyev et al., 2016). It is also worth noting that sometimes different constitutive models may lead to the same qualitative wave pattern, as it is shown by Eremeyev et al. (2018) for antiplane wave propagation within the Gurtin-Murdoch surface elasticity or considering the Toupin-Mindlin strain-gradient elasticity models.

When stress concentration is investigated in microstructured media, it appears that all contributions available in the literature deal with static or steady-state propagating problems. Zhang et al. (1998) give the full-field solution and stress intensity factors for the static Mode III crack problem (antiplane deformation) in a reduced CS material with three material parameters (although only two affect the antiplane behaviour). The general solution for indeterminate CS materials is given in Radi (2008). Later, the problem of steady-state Mode III crack propagation has been investigated by Mishuris et al. (2012) and Morini et al. (2014, 2013). Georgiadis (2003) appears to be the first and only contribution considering dynamic stress concentration in a straight semi-infinite crack in the presence of microstructure, although the latter is accounted for through the simpler grade two strain gradient theory. Besides, the classical linear elastic fracture mechanics field, with time-harmonic variation, is considered in the far field as the forcing term.

In this paper, a travelling wave loading, applied in the form of shear reduced tractions at the crack faces, is considered as the forcing term. As a result, a complicated wave pattern appears, which differs significantly from the classical solution given in Freund (1990). This loading condition may be used as a building block to address, by means of superposition, any wave propagation problem in a cracked CS half-space. Resonance is triggered when the applied loading is fed into the cracktip at Rayleigh speed. Elastodynamic stress intensity factors are given, which generalize the corresponding results presented in Radi (2008) for the static regime. They incorporate the effect of the applied loading frequency and thereby account for the interplay of the diffracted waves.

\section{Antiplane couple stress elasticity}

Let us consider a Cartesian co-ordinate system $\left(0, x_{1}, x_{2}, x_{3}\right)$, such that the rectilinear crack occupies the semi-infinite line $x_{1}<0$, Fig. 1 . The indeterminate theory of CS elasticity adopted in the present study provides the following kinematical (compatibility) conditions (Koiter, 1964, Eqs. (4.9)) for the strain tensor

$$
\boldsymbol{\varepsilon}=\text { Sym grad } \boldsymbol{u},
$$

for the rotation vector

$$
\boldsymbol{\varphi}=\frac{1}{2} \operatorname{curl} \boldsymbol{u},
$$

and for the torsion-flexure tensor

$$
\chi=\operatorname{grad} \varphi .
$$

We observe that, through Eq. (3), micro-rotations are determined by the macro-motion, which feature makes the CS theory a restriction of the micropolar theory. Under antiplane shear deformation, the displacement field $\boldsymbol{u}=\left(u_{1}, u_{2}, u_{3}\right)$ is completely 


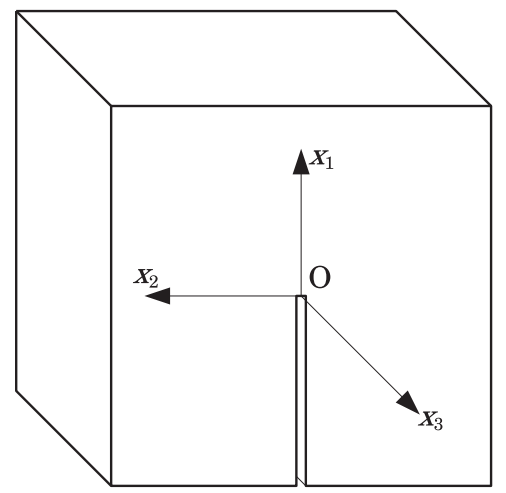

Fig. 1. Semi-infinite rectilinear crack $x_{1}<0$ in a half-space under antiplane deformation along $x_{3}$.

defined by the out-of-plane component $u_{3}=u_{3}\left(x_{1}, x_{2}, x_{3}, t\right)$. The nonzero components of the strain, rotation and of the flexure-torsion tensors become

$$
\begin{aligned}
& \varepsilon_{13}=\frac{1}{2} u_{3,1}, \quad \varepsilon_{23}=\frac{1}{2} u_{3,2}, \\
& \varphi_{1}=\frac{1}{2} u_{3,2}, \quad \varphi_{2}=-\frac{1}{2} u_{3,1}, \\
& \chi_{11}=-\chi_{22}=\frac{1}{2} u_{3,12}, \quad \chi_{21}=-\frac{1}{2} u_{3,11}, \quad \chi_{12}=\frac{1}{2} u_{3,22} .
\end{aligned}
$$

Hereinafter, a subscript comma denotes partial differentiation, e.g. $u_{3, i}=\partial u_{3} / \partial x_{i}$.

The Cauchy stress tensor $\boldsymbol{t}$ is decomposed into its symmetric and skew-symmetric parts, respectively $\boldsymbol{\sigma}$ and $\boldsymbol{\tau}$,

$$
\boldsymbol{t}=\boldsymbol{\sigma}+\boldsymbol{\tau}, \quad \boldsymbol{\sigma}=\operatorname{Sym} \boldsymbol{t}, \quad \boldsymbol{\tau}=\operatorname{Skw} \boldsymbol{t} .
$$

In addition, the deviatoric part of the couple stress tensor, $\boldsymbol{\mu}$, is introduced as being work-conjugated to $\chi^{T}$ (Koiter, 1964 , Eq. (2.22)). Indeed, the CS theory is named indeterminate after the observation that the first invariant of the couple-stress tensor, i.e. $\operatorname{tr} \boldsymbol{\mu}=\mu_{11}+\mu_{22}+\mu_{33}$, rests indeterminate and therefore it may be set equal to zero without loss of generality.

At any point of a smooth surface we may specify the reduced force traction vector $\boldsymbol{p}$ and the tangential part of the couple stress traction vector $\boldsymbol{q}$ (Koiter, 1964, Eqs. (3.5-6))

$$
\boldsymbol{p}=\boldsymbol{t}^{T} \boldsymbol{n}+\frac{1}{2} \operatorname{grad} \mu_{n n} \times \boldsymbol{n}, \quad \boldsymbol{q}=\boldsymbol{\mu}^{T} \boldsymbol{n}-\mu_{n n} \boldsymbol{n},
$$

where we have $\mu_{n n}=\boldsymbol{n} \cdot \boldsymbol{\mu n}=\boldsymbol{q} \cdot \boldsymbol{n}$. In particular, at the bottom/top crack face $x_{2}=0^{\mp}$, it is $\boldsymbol{n}= \pm(0,1,0)$ and, according to Eq. (5), the out-of-plane component of the reduced force traction and the in-plane components of the couple stress traction read, respectively,

$$
p_{3}= \pm\left(t_{23}+\frac{1}{2} \mu_{22,1}\right), \quad q_{1}= \pm \mu_{21}, \quad q_{2}=0 .
$$

The conditions of dynamic equilibrium of forces and moments read (Koiter, 1964, Eqs. (2.7) and (2.9))

$$
\begin{aligned}
& \sigma_{13,1}+\sigma_{23,2}+\tau_{13,1}+\tau_{23,2}=\rho \ddot{u}_{3}, \\
& \mu_{11,1}+\mu_{21,2}+2 \tau_{23}=J \ddot{\varphi}_{1}, \\
& \mu_{12,1}+\mu_{22,2}-2 \tau_{13}=J \ddot{\varphi}_{2},
\end{aligned}
$$

where $\mathrm{e}$ is the mass density and $J$ is the rotational inertia. Within the framework of linear deformation, the total strain $\varepsilon$ and the curvature $\chi$ are connected to the stress and to the couple stress through the isotropic constitutive relations

$$
\boldsymbol{\sigma}=2 G \boldsymbol{\varepsilon}+\Lambda(\operatorname{tr} \boldsymbol{\varepsilon}) \mathbf{1}, \quad \boldsymbol{\mu}=2 G \ell^{2}\left(\chi^{T}+\eta \chi\right)
$$

where $\Lambda$ and $G>0$ take up the role of Lamé moduli, $\mathbf{1}$ is the identity tensor, $\ell>0$ is a characteristic length and $-1<\eta<1$ is a dimensionless number similar to Poisson's ratio. Clearly, classical elasticity is retrieved taking $\ell=0$ and $J=0$. We observe that the contribution of $\Lambda$ is immaterial for antiplane deformations, cf. Zhang et al. (1998, Eqs. (8-9)). Besides, the second equation in (8) differs from Koiter (1964, Eqs. (4.7)) by a factor 2, which is incorporated in $\ell$. The material parameters $\ell$ and $\eta$ depend on the microstructure and can be connected to the material characteristic length in bending, $\ell_{b}$, and in torsion, $\ell_{t}$, through

$$
\ell_{b}=\ell / \sqrt{2}, \quad \ell_{t}=\ell \sqrt{1+\eta}
$$


Values of $\ell_{b}$ and $\ell_{t}$ may be found in Lakes (1986); Nakamura and Lakes (1995) and, as an example, for polyurethane foam we have

$$
\ell=0.462 \mathrm{~mm}, \quad \eta=0.797
$$

The limiting value $\eta=-1$ corresponds to a vanishing characteristic length in torsion, which is typical of polycrystalline metals. Clearly, the definitions (9) show that $\ell_{t}=\ell_{b}$ for $\eta=-\frac{1}{2}$ and $\ell_{t}=\ell=\sqrt{2} \ell_{b}$ for $\eta=0$, the latter situation being the strain gradient effect considered in Zhang et al. (1998). For the limiting value $\eta=1$, the constitutive Eq. (8) provides a symmetric couple stress tensor and, consequently, the present theory reduces to the modified couple stress theory of elasticity introduced in Yang et al. (2002). Indeed, the simplified couple stress theory involves only the material length $\ell$ for $\ell_{b}=\ell_{t} / 2=\ell / \sqrt{2}$.

The constitutive equations (8), together with the kinematic relations (1)-(4), give stress and couple stress in terms of displacement

$$
\begin{aligned}
& \sigma_{13}=G u_{3,1}, \quad \sigma_{23}=G u_{3,2}, \\
& \mu_{11}=-\mu_{22}=G \ell^{2}(1+\eta) u_{3,12}, \quad \mu_{21}=G \ell^{2}\left(u_{3,22}-\eta u_{3,11}\right), \\
& \mu_{12}=-G \ell^{2}\left(u_{3,11}-\eta u_{3,22}\right) .
\end{aligned}
$$

We observe that the skew-symmetric part $\boldsymbol{\tau}$ of the total stress tensor $\boldsymbol{t}$ is determined by rotational equilibrium. Indeed, introduction of Eq. (10) into Eqs. (7b) and (7c) yields

$$
\tau_{13}=-\frac{1}{2} G \ell^{2} \Delta u_{3,1}+\frac{J}{4} \ddot{u}_{3,1}, \quad \tau_{23}=-\frac{1}{2} G \ell^{2} \Delta u_{3,2}+\frac{J}{4} \ddot{u}_{3,2},
$$

which correspond to Eqs. (9) of Mishuris et al. (2012). Here, $\Delta$ denotes the 2-D Laplace operator.

\section{Time-harmonic analysis}

It is found expedient to introduce the reference length $\lambda \ell$ and the reference time $T=\ell / c_{s}$ along with the dimensionless co-ordinates $\left(\xi_{1}, \xi_{2}, \xi_{3}\right)=(\lambda \ell)^{-1}\left(x_{1}, x_{2}, x_{3}\right)$ and the dimensionless time $\tau=t / T$. Here, $c_{s}=\sqrt{G / \rho}$ is the shear wave speed of classical elastic media and $\lambda$ is defined in the following. We consider a shear traction wave applied to the crack faces and zero micropolar stress

$$
p_{3}\left(\xi_{1}, 0^{ \pm}, \tau\right)= \pm G \tau_{0} \exp \left[\imath\left(k \xi_{1}+\Omega \tau\right)\right], \quad q_{1}\left(\xi_{1}, 0^{ \pm}, \tau\right)=0, \quad \xi_{1}<0,
$$

where 1 is the imaginary unit and $\Omega=\omega T>0$ the dimensionless (time) frequency. Here, $k$ denotes the dimensionless (spatial) wavenumber and it is a complex number with non-positive imaginary part, i.e. $\Im(k) \leq 0$, to warrant propagation/decay as $\xi_{1} \rightarrow-\infty$. In fact, the limiting case $\Im(k)=0$ corresponds to a propagating wave with phase velocity

$$
c=\frac{\Omega}{k} \lambda c_{s}
$$

When $\Re(k) \lessgtr 0$, the applied wave is impinging upon/moving out of the crack-tip. In the special case $\Re(k)=0$, a harmonic (in time) loading, exponentially decaying along the crack, is considered. We observe that, in the general case, $\tau_{0}=\tau_{0}(k, \Omega)$ and this problem may be used as a building block to solve any harmonic wave propagation problem in a cracked couple stress half-space in antiplane deformation.

Assuming the same time-harmonic variation for the out-of-plane displacement as in the applied wave (12)

$$
u_{3}\left(\xi_{1}, \xi_{2}, \tau\right)=\ell w\left(\xi_{1}, \xi_{2}\right) \exp \imath \Omega \tau,
$$

and substituting Eqs. (10a)-(11) into (7)-(7c), we get the metaharmonic PDE (Georgiadis and Velgaki, 2003, (19)) for the function $w$ :

$$
\Delta \Delta w-2\left(1-h_{0}^{2} \Omega^{2}\right) \lambda^{2} \Delta w-2 \Omega^{2} \lambda^{4} w=0,
$$

where $\Delta w=w_{, 11}+w_{, 22}$ and we have let the dimensionless parameter (Mishuris et al., 2012)

$$
h_{0}=\frac{h}{\ell}, \quad \text { with } \quad h=\frac{1}{2} \sqrt{\frac{J}{\rho}} .
$$

We observe that $h$ is proportional to the dynamic characteristic length introduced in Shodja et al. (2015). This generalized bi-harmonic equation can be easily factored

$$
\left(\Delta+\delta^{2}\right)(\Delta-1) w=0,
$$

where we have let the positive dimensionless parameter

$$
\delta=\frac{1}{\sqrt{2} \Omega}\left[\sqrt{\left(1-h_{0}^{2} \Omega^{2}\right)^{2}+2 \Omega^{2}}-1+h_{0}^{2} \Omega^{2}\right],
$$


and, for convenience, we have chosen the scaling factor $\lambda$ as to have 1 in the second factor of Eq. (15)

$$
\lambda=\sqrt{\frac{\delta}{\sqrt{2} \Omega}} .
$$

We observe that $\lambda$ is a strictly monotonic increasing (decreasing) function of $\Omega$ inasmuch as $h_{0} \gtrless h_{0 c r}=1 / \sqrt{2}$, while $h_{0}=$ $h_{0 c r}$ lends $\delta=\Omega / \sqrt{2}$ and $\lambda \equiv h_{0 c r}$. The latter situation corresponds to classical elasticity, for then the governing equation (15) becomes

$$
\left(\Delta+\frac{1}{2} \Omega^{2}\right)(\Delta-1) w=0
$$

and the former factor corresponds to the Helmoltz equation that governs shear waves within classical elasticity (Freund, 1990, Eq. (2.2.4)). In particular, in the static limit $\Omega \rightarrow 0$, we get $\delta \rightarrow 0$ and Eq. (17) boils down to

$$
\Delta(\Delta-1) w=0
$$

that recovers the governing equation for the static regime considered in Radi (2008, Eq. (10)) and in Zhang et al. (1998, Eq. (11)).

Using Eqs. (1), (8), (10b) and (11) into the first of Eq. (6), the corresponding loading conditions (12) becomes

$$
\left(1-\delta^{2}\right) w_{, 2}-\left[(2+\eta) w_{, 11}+w_{, 22}\right]_{, 2}=2 \lambda^{3} \tau_{0} \exp \imath k \xi_{1}, \quad \xi_{1}<0, \xi_{2}=0^{+} .
$$

Besides, the skew-symmetric character of Mode III requires

$$
w\left(\xi_{1}, 0\right)=0, \quad \xi_{1}>0
$$

$$
q_{1}\left(\xi_{1}, 0\right)=0, \quad \xi_{1}>0 .
$$

Therefore, the condition of zero micropolar traction $q_{1}\left(\xi_{1}, 0\right)=0$ stands along the whole crack line and, in light of Eqs. (6) and (10b), it gives

$$
w_{, 22}-\eta w_{, 11}=0, \quad \xi_{2}=0 .
$$

We note that the material parameter $\eta$ only appears in the boundary conditions (18) and (20). It is worth emphasizing that, within this framework, the single boundary condition of classical antiplane elasticity cannot be retrieved. However, upon taking $\eta=0$ in addition to $h_{0}=h_{0 c r}$, the classical shear wave solution is recovered, for it satisfies both boundary conditions.

\subsection{Rayleigh waves for antiplane deformation}

Planar shear waves travelling in the bulk of the material have been considered in Mishuris et al. (2012, Section 2.1). Here, similarly to Ottosen et al. (2000) and Georgiadis and Velgaki (2003), we look at localized solutions

$$
u_{3}\left(\xi_{1}, \xi_{2}, \tau\right)=\ell W\left(\xi_{2}\right) \exp \left[l\left(m \xi_{1}+\Omega \tau\right)\right]
$$

where $W\left(\xi_{2}\right)$ decays fast enough away from the crack line and we assume $m$ to be real. Then, Eq. (15) governing harmonic motion for antiplane deformation gives

$$
W^{\prime \prime \prime \prime}+\left(\delta^{2}-2 m^{2}-1\right) W^{\prime \prime}+\left(m^{2}+1\right)\left(m^{2}-\delta^{2}\right) W=0
$$

which admits the solution

$$
W\left(\xi_{2}\right)=C_{1} \exp \left[-\alpha(m) \xi_{2}\right]+C_{2} \exp \left[-\beta(m) \xi_{2}\right]
$$

where

$$
\alpha(s)=\sqrt{s^{2}-\delta^{2}}, \quad \beta(s)=\sqrt{s^{2}+1}
$$

The constants $C_{1}$ and $C_{2}$ are determined imposing homogeneous boundary conditions on the crack surface, which amounts to the vanishing of reduced force and couple stress traction at $\xi_{2}=0$,

$$
\left\{\begin{array}{l}
W^{\prime}(0)+\frac{1}{1-\delta^{2}}\left[(2+\eta) m^{2} W^{\prime}(0)-W^{\prime \prime \prime}(0)\right]=0 \\
{[1.1 e x] W^{\prime \prime}(0)+\eta m^{2} W(0)=0}
\end{array}\right.
$$

Plugging Eq. (21) into the boundary conditions (23) yields the following linear system for the constants $C_{1}$ and $C_{2}$

$$
\left\{\begin{array}{l}
\alpha(m)\left[(\eta+1) m^{2}+1\right] C_{1}+\beta(m)\left[(\eta+1) m^{2}-\delta^{2}\right] C_{2}=0 \\
{[1.1 e x]\left[(\eta+1) m^{2}-\delta^{2}\right] C_{1}+\left[(\eta+1) m^{2}+1\right] C_{2}=0}
\end{array},\right.
$$




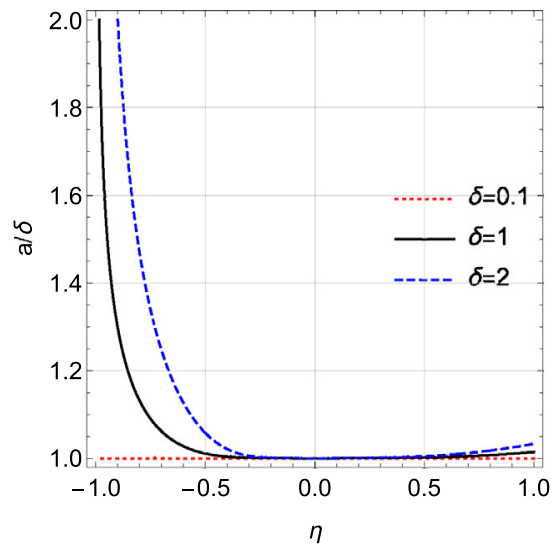

(a)

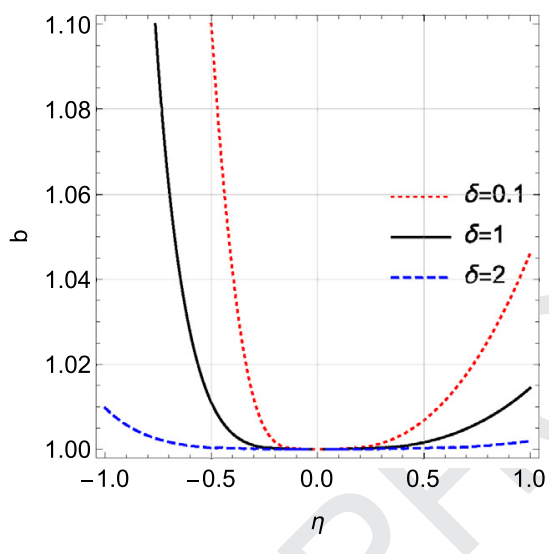

(b)

Fig. 2. Location of the real root $a$ (a) of the purely imaginary root $\mathrm{l} b$ (b) as a function of $\eta$ for $\delta=0.1$ (red, dotted), $\delta=1$ (black, solid) and $\delta=2$ (blue, dashed). (For interpretation of the references to colour in this figure legend, the reader is referred to the web version of this article.)

which admits non-trivial solutions if and only if the following secular equation is satisfied

$$
R(m)=0,
$$

where

$$
R(s)=\alpha(s)\left[(\eta+1) s^{2}+1\right]^{2}-\beta(s)\left[(\eta+1) s^{2}-\delta^{2}\right]^{2} .
$$

The function $R(s)$ is the Rayleigh wave function for antiplane deformation in couple stress elastic materials. For the selected branch cuts, the function $R(s)$ is single valued and analytic in the cut $s$-plane. In the antiplane problem of classical elasticity, the Rayleigh function is simply $\alpha(s)$ (Harris, 2001, Eq. (5.96)), that possesses two branch points, which correspond to bulk shear waves, yet no real roots, i.e. no Rayleigh waves are supported. In contrast, for the considered range of variation of the parameters $\delta$ and $\eta$, Eq. (24) admits three complex conjugated pairs of order- 1 roots, namely two real roots $s= \pm a$, two purely imaginary roots $s= \pm l b$ and the pair of complex roots $s= \pm s_{3}$, which may fall out of the physical Riemann sheet. The location of the roots $a$ and $1 b$ against the parameters $\eta$ and $\delta$ is presented in Fig. 2. There, it can be observed that $a \geq \delta$, $b \geq 1$ and yet they sit very close to the branch points, especially for $\eta>-1 / 2$. In particular, equality holds for $\eta=0$, for which value roots become branch points (order $1 / 2$ ). In the static limit, branch points collapse at the origin (i.e. $\delta=0$ ) that becomes an order 1 root.

The pair of imaginary roots $\pm 1 b$ is here connected to the strain-gradient effect. In general, it is associated to a fourthorder governing equation and it accounts for the edge-effect in shell theories Kaplunov and Nobili (2017) or for evanescent modes in supported plates (Nobili et al., 2017).

The real root $a>0$ of the dispersion relation (24) provides the Rayleigh wave speed $c_{R}$ according to Eq. (13)

$$
\frac{c_{R}}{c_{s}}=\frac{1}{a} \sqrt{\frac{\Omega \delta}{\sqrt{2}}}=\frac{\sqrt{\sqrt{\left(1-h_{0}^{2} \Omega^{2}\right)^{2}+2 \Omega^{2}}-1+h_{0}^{2} \Omega^{2}}}{\sqrt{2} a} .
$$

In the low-frequency limit, $\Omega \rightarrow 0^{+}$, we have $a \sim \delta \sim \Omega / \sqrt{2}$, whence $c_{R} \rightarrow c_{S}$ and Rayleigh waves collapse into classical shear waves. In the special case $\eta=0$, the roots $a$ and $1 b$ collapse into the branch points $\delta$ and 1 , respectively, while $s_{3}$ falls out of the physical sheet. In this case, bulk shear waves are obtained, whose speed $\tilde{c}$ is found replacing $a$ with $\delta$ in Eq. (26) and using Eq. (16) (cf. Mishuris et al., 2012, Eq. (14))

$$
\frac{\tilde{c}}{c_{s}}=\frac{\sqrt{\sqrt{\left(1-h_{0}^{2} \Omega^{2}\right)^{2}+2 \Omega^{2}}+1-h_{0}^{2} \Omega^{2}}}{\sqrt{2}} .
$$

In fact, we have $\alpha(\delta)=0=\beta(\imath)$ and the solution (21) is no longer decaying away from the crack line. As it is usually the case, Rayleigh waves occur at speed slightly below that of bulk shear waves, i.e. $c_{R}<\tilde{c}$ (Destrade et al., 2016).

In the special case $h_{0}=h_{0 c r}$ and $\eta=0$, the situation of classical antiplane elasticity is retrieved, which does not support Rayleigh waves: indeed these collapse into non-dispersive shear bulk waves, i.e. $c_{R}=\tilde{c} \equiv c_{s}$, see Fig. $3 c$.

Dispersion curves for the relative Rayleigh-wave speed $c_{R} / c_{s}$ for different values of the microstructural parameters $h_{0}$ and $\eta$ are plotted in Fig. 3. As anticipated, in the long-wave low-frequency limit, the antiplane Rayleigh-wave speed $c_{R}$ recovers the shear wave speed of classical elastic media $c_{s}$. We observe that, in the absence of rotational inertia (i.e. $h_{0}=0$ ), the Rayleigh-wave speed $c_{R}$ grows monotonically with the wavenumber (Fig. 3a) and waves are thereby dispersive, as already 




(a) $h_{0}=0$

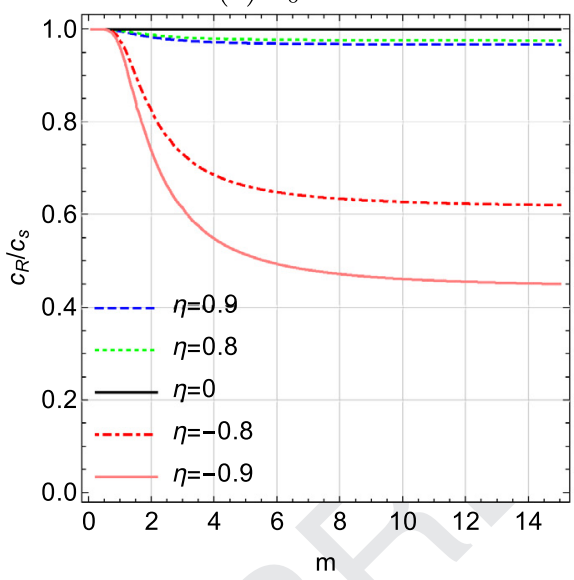

(c) $h_{0}=h_{0 c r}$

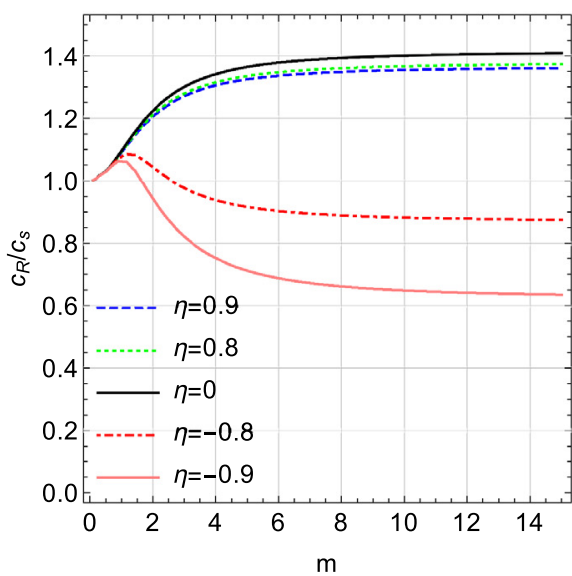

(b) $h_{0}=0.5$

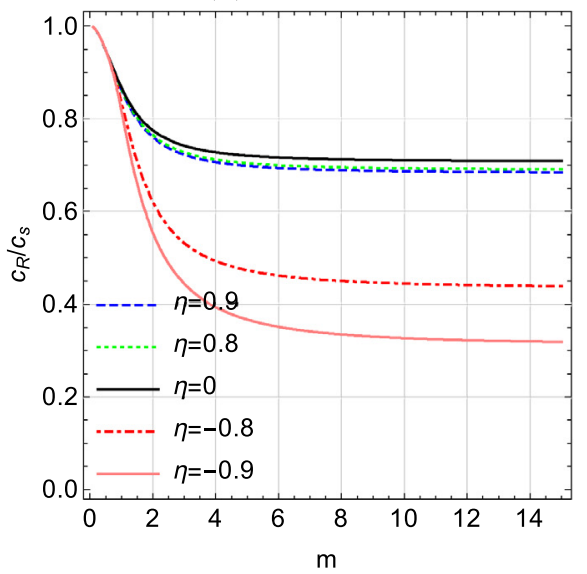

(d) $h_{0}=1$

Fig. 3. Dispersion curves for the dimensionless Rayleigh wave speed $c_{R} / c_{s}$ (the curve $\eta=0$ corresponds to the bulk wave speed $\tilde{c} / c_{s}$ ).

found in Graff and Pao (1967) and Ottosen et al. (2000) for plane-strain Rayleigh waves. Besides, $c_{R}>c_{S}$, which is physically unrealistic (Shodja et al., 2015). In contrast, for $h_{0}>0$, the Rayleigh-wave speed quickly asymptotes a finite limit and the dispersive character of propagation is really restricted to low wavenumbers. Propagation turns perfectly non-dispersive, as in classical elasticity, with speed $c_{R} \equiv c_{s}$, for $h_{0}=h_{0 c r}$ and $\eta=0$, see Fig. 3c. For $h_{0}>h_{0 c r}$ and $\eta \neq 0$ or for $h_{0}>0$ and $\eta$ close enough to -1 , the Rayleigh-wave speed becomes a decreasing function of wavenumber. This behaviour is discussed in Georgiadis and Velgaki (2003), in the context of plane-strain Rayleigh wave propagation, with reference to experimental results and lattice theories.

Fig. 4 superposes dispersion curves for the group velocity $c_{g}$

$$
\frac{c_{g}}{c_{s}}=\frac{\lambda^{2}}{\frac{\mathrm{d} m}{\mathrm{~d} \Omega} \lambda-m \frac{\mathrm{d} \lambda}{\mathrm{d} \Omega}},
$$

over the corresponding dispersion curve for the Rayleigh wave phase velocity $c_{R}$. Dispersion is termed anomalous when $c_{g}>c_{R}$ and normal otherwise (Achenbach, 1984, Section 6.5). Anomalous dispersion is met for $h_{0}=0$ at any $\eta$ and for $0<h_{0}<h_{0 c r}$ and $\eta$ close to 1 . This condition is related to energy propagating faster than the wavelets which build up at the front of the group and slowly move to the back until they disappear, see Gourgiotis et al. (2013, Section 2) in the context of gradient elasticity.

\section{Analysis in the frequency domain}

We adopt Fourier transforms to recast the problem in the frequency domain. Owing to the skew-symmetry of the problem, only either half-plane, say $\xi_{2}>0$, needs to be considered. The full-range Fourier transform along $\xi_{1}$ is defined as 


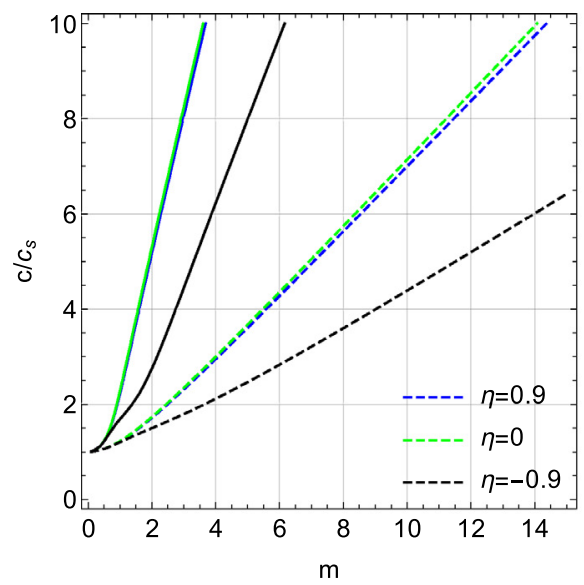

(a) $h_{0}=0$

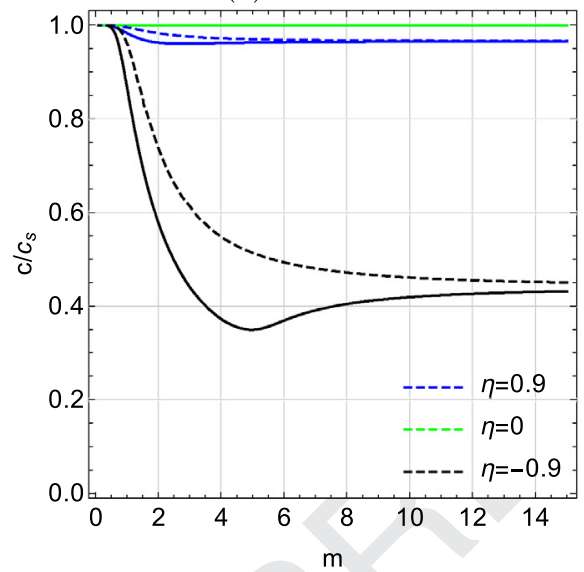

(c) $h_{0}=h_{0 c r}$

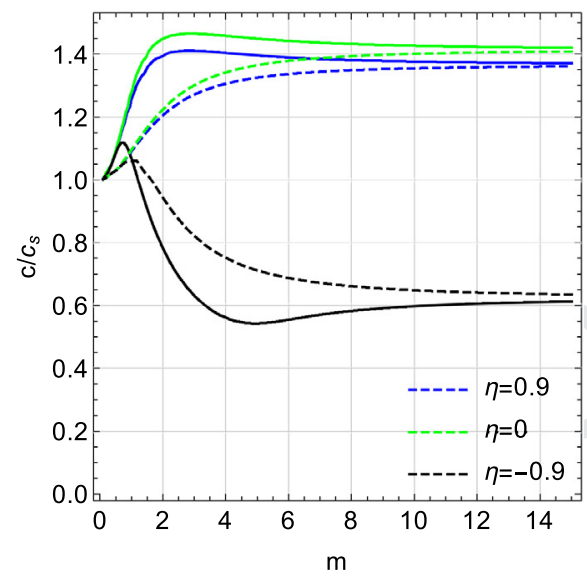

(b) $h_{0}=0.5$

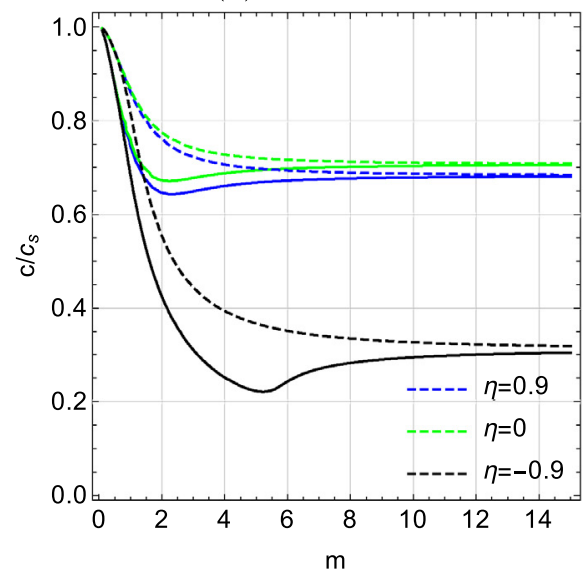

(d) $h_{0}=1$

Fig. 4. Relative group velocity $c_{g} / c_{s}$ (solid) and Rayleigh wave speed $c_{R} / c_{s}$ (dashed) as a function of wavenumber (the curve $\eta=0$ corresponds to the bulk wave speed $\tilde{c} / c_{s}$ ).

\section{(Noble, 1958; Roos, 1969)}

$$
\bar{w}\left(s, \xi_{2}\right)=\int_{-\infty}^{\infty} w\left(\xi_{1}, \xi_{2}\right) \exp \left(\imath \xi_{1} s\right) \mathrm{d} \xi_{1},
$$

while Fourier inversion formula gives

$$
w\left(\xi_{1}, \xi_{2}\right)=(2 \pi)^{-1} \int_{\mathscr{L}} \bar{w}\left(s, \xi_{2}\right) \exp \left(-\imath \xi_{1} s\right) \mathrm{d} s .
$$

In the inverse transform, the integration path $\mathscr{L}$ is obtained by deformation of the real interval, as described in the following. The complex plane $\mathbb{C}$ is split into two domains, $\mathbb{C}=\mathcal{D}^{+} \cup \mathcal{D}^{-}$, respectively lying on and above and on and below the integration path $\mathscr{L}$.

Let us define the half-range Fourier transforms

$$
\bar{w}^{-}(s)=\int_{-\infty}^{0} w\left(\xi_{1}, 0\right) \exp \left(\imath \xi_{1} s\right) \mathrm{d} \xi_{1}, \quad \bar{p}_{3}^{+}(s)=\int_{0}^{\infty} p_{3}\left(\xi_{1}, 0\right) \exp \left(\imath \xi_{1} s\right) \mathrm{d} \xi_{1} .
$$

Taking the full-range Fourier transform of Eq. (15) gives

$$
\bar{w}_{, 2222}-\left(2 s^{2}+1-\delta^{2}\right) \bar{w}_{, 22}+\left(s^{2}+1\right)\left(s^{2}-\delta^{2}\right) \bar{w}=0,
$$

which admits the solution (21)

$$
\bar{w}\left(s, \xi_{2}\right)=C_{1} \exp \left[-\alpha(s) \xi_{2}\right]+C_{2} \exp \left[-\beta(s) \xi_{2}\right],
$$

where $C_{1}$ and $C_{2}$ are complex-valued functions of $s$. 


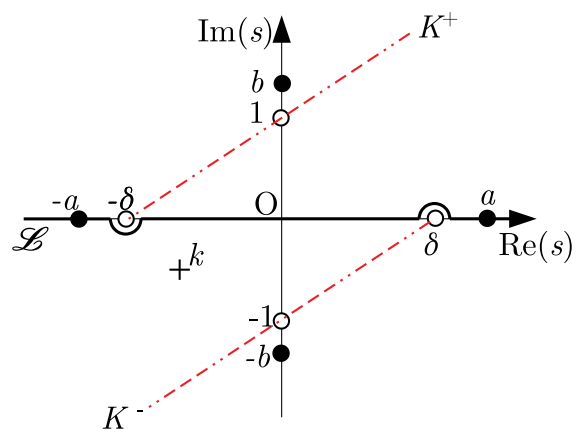

Fig. 5. Branch cuts $\mathcal{K}^{ \pm}$(red dash-double dot lines), zeros (black dots), branch points (circles), applied traction wavenumber (cross) and integration path $\mathscr{L}$ (solid curve) in the complex plane $\mathbb{C}=\mathcal{D}^{+} \cup \mathcal{D}^{-}$. The domains $\mathcal{D}^{ \pm}$stand on and above (under) the integration path $\mathscr{L}$, respectively. (For interpretation of the references to colour in this figure legend, the reader is referred to the web version of this article.)

Here $\alpha(s)$ and $\beta(s)$ are defined as in (22) but now $s$ is generally complex and we need to specify the branch in the square root so that (27) is bounded as $\xi_{2} \rightarrow+\infty$. The branch cuts for $\alpha(s)$, denoted by $\mathcal{K}^{\mp}$, are straight and connect, respectively, the branch points $s= \pm \delta$ to $\infty$ in the direction $\mp(\delta+\imath)$ and thereby they pass through $s=\mp l$, as shown in Fig. 5. They may be parametrized as

$$
\mathcal{K}^{\mp}=\{s(t)= \pm \delta \mp(\delta+\imath) t, \quad t>0\} .
$$

In likewise fashion, the branch cuts for $\beta(s)$ rest inside $\mathcal{K}^{\mp}$ and connect the branch points $s= \pm \imath$ to $\mp(\delta+\imath) \infty$, i.e. they have the parametrization (28) with $t>1$. Square root is made definite by letting $\alpha(s)$ and $\beta(s)$ tend to $|s|$ as $s \rightarrow \infty$ along the real axis (see Noble, 1958, p.10). With such definitions, the square roots in $\alpha(s)$ and $\beta(s)$ are defined so as to have positive or zero real part (respectively decaying and propagating solutions) when $s$ is real. Indeed, for $s=s_{1} \in \mathbb{R}$, it is

$$
\alpha\left(s_{1}\right)=\left\{\begin{array}{ll}
l \sqrt{\delta^{2}-s_{1}^{2}}, & \left|s_{1}\right|<\delta \\
\sqrt{s_{1}^{2}-\delta^{2}}, & s_{1} \geq \delta
\end{array}, \quad \beta\left(s_{1}\right)=\sqrt{s_{1}^{2}+1} .\right.
$$

The full-range transform of the reduced traction vector at the l.h.s of Eq. (18) is

$$
\bar{p}_{3}\left(s, \xi_{2}\right)=\frac{G}{2 \lambda^{3}}\left[\left((2+\eta) s^{2}+1-\delta^{2}\right) \bar{w}_{, 2}-\bar{w}_{, 222}\right] .
$$

The minus half-range Fourier transform of the first boundary condition (12) reads

$$
\bar{p}_{3}^{-}(s, 0)=-l \frac{G \tau_{0}}{s+k}
$$

and the plus transform of Eq. (19a) lends

$$
\bar{w}^{+}(s, 0)=0 \text {, }
$$

while the full-range transform of Eq. (20) gives

$$
\bar{w}_{, 22}(s, 0)+\eta s^{2} \bar{w}(s, 0)=0 .
$$

Plugging the solution (27) into Eq. (33) gives a connection between $C_{1}$ and $C_{2}$

$$
C_{1}=-\frac{(\eta+1) s^{2}+1}{(\eta+1) s^{2}-\delta^{2}} C_{2}
$$

Thus, making use of the general solution (27) and of the connection (34), we find for the full-range Fourier transform of the traction vector (30)

$$
\frac{G\left(1+\delta^{2}\right)}{2 \lambda^{3}\left[(\eta+1) s^{2}-\delta^{2}\right]} K(s) C_{2}=\bar{p}_{3}^{+}(s, 0)-\imath \frac{G \tau_{0}}{s+k},
$$

where

$$
K(s)=\left(1+\delta^{2}\right)^{-1} R(s),
$$

and $R(s)$ is the Rayleigh function introduced in Eq. (25). The kernel function $K(s)$ is even and it obeys the reflection principle

$$
K\left(s^{*}\right)=K(s)^{*},
$$

where a superscript asterisk denotes complex conjugation, i.e. $s^{*}=\Re(s)-\imath \Im(s)$. As a consequence, $K(s)$ is real-valued on the real axis. 
In light of Eqs. (27), (32) and (34), we get for the displacement $\bar{w}(s, 0)$

$$
-\frac{1+\delta^{2}}{(\eta+1) s^{2}-\delta^{2}} C_{2}=\bar{w}^{-}(s, 0) \text {. }
$$

Solving Eq. (38) for $C_{2}$ and substituting into Eq. (35) yields the inhomogeneous Wiener-Hopf functional equation

$$
G^{-1} \bar{p}_{3}^{+}(s, 0)=-\frac{1}{2 \lambda^{3}} K(s) \bar{w}^{-}(s, 0)+l \frac{\tau_{0}}{s+k} .
$$

\subsection{Asymptotic behaviour}

The asymptotic behaviour of the out of plane reduced traction and displacement at the crack tip is expected to be the same as what is obtained in the stationary crack problem under quasi-static loading, respectively (Radi, 2008, Eqs. (39) and (40))

$$
\begin{aligned}
& p_{3}\left(\xi_{1}, 0\right)=O\left(\xi_{1}^{-3 / 2}\right), \quad \text { as } \xi_{1} \rightarrow 0^{+}, \\
& w\left(\xi_{1}, 0\right)=O\left(\left(-\xi_{1}\right)^{3 / 2}\right), \quad \text { as } \xi_{1} \rightarrow 0^{-} .
\end{aligned}
$$

Consequently, the Tauberian theorem for the Fourier transform gives (Roos, 1969)

$$
\bar{p}_{3}^{+}(s, 0)=O\left(s^{1 / 2}\right), \quad \text { and } \quad \bar{w}^{-}(s, 0)=O\left(s^{-5 / 2}\right), \quad \text { as }|s| \rightarrow \infty .
$$

\subsection{Radiation condition}

As discussed in (Noble, 1958, Section 1.5), application of the W-H technique requires a strip of regularity, which is warranted when a small imaginary part for $\delta$ is assumed such that $\delta^{2}=\delta_{1}^{2}-\imath \epsilon \delta_{1}$. Nonetheless, the limiting situation $\epsilon=0$ is still accessible provided that Sommerfeld's radiation condition is enforced. This demands that elastic waves transfer energy from the loading zone to infinity and not vice versa. In particular, along the crack surface, energy is carried away by Rayleigh waves, travelling in the negative $\xi_{1}$-direction with the speed $c_{R}$ defined in Eq. (26). Accordingly, proceeding as in Section 3.1, we have

$$
w\left(\xi_{1}, 0\right)=A \exp \left(\imath a \xi_{1}\right)+O\left(\xi_{1}^{-\rho}\right), \quad \text { as } \xi_{1} \rightarrow-\infty,
$$

where $A$ is a constant and $\rho>0$ warrants decay. The corresponding minus Fourier transform can be split as

$$
\bar{w}^{-}(s, 0)=\int_{-\infty}^{-M}+\int_{-M}^{0} w\left(\xi_{1}, 0\right) \exp \left(\imath \xi_{1}\right) \mathrm{d} \xi_{1},
$$

for any large positive constant $M$. We observe that the second integral is an entire function, for it has no singular points in the complex plane. Consequently, all singular points of the function $\bar{w}^{-}(s, 0)$ come with the first integral

$$
\int_{-\infty}^{M} \exp \left[-l(s+a) \xi_{1}\right] \mathrm{d} \xi_{1}=\frac{l}{s+a} \exp [\imath M(s+a)],
$$

which indeed brings the singular point $s=-a$. Therefore, it follows that

$$
\bar{w}^{-}(s, 0)=(s+a)^{-1}, \quad \text { as } s \rightarrow-a .
$$

Along every other direction, different from the negative $\xi_{1}$-axis, Sommerfeld's radiation condition for the governing Eq. (14) requires, in polar co-ordinates $(r, \vartheta), r=\left(\xi_{1}^{2}+\xi_{2}^{2}\right)^{1 / 2}$, (Noble, 1958, Section 1.5)

$$
\frac{\partial w}{\partial r}+\imath \delta w=o\left(\frac{1}{\sqrt{r}}\right), \quad \text { as } r \rightarrow \infty \quad(\vartheta \neq \pm \pi),
$$

uniformly in 9 . Such behaviour should be recovered by the present solution, although the field equation (12) is not of the classical Helmholtz type (Georgiadis and Vardoulakis, 1998). Condition (42) is compatible with the following asymptotic behaviour for the displacement along radial lines

$$
w(r, \vartheta)=O\left(r^{-1 / 2} \exp (-\imath r \delta)\right), \quad \text { as } r \rightarrow \infty \quad(\vartheta \neq \pm \pi),
$$

and in particular, for $\vartheta=\pi / 2$, the $\xi_{2}$-axis is considered

$$
w\left(0, \xi_{2}\right)=O\left(\xi_{2}^{-1 / 2} \exp \left(-\imath \xi_{2} \delta\right)\right), \quad \text { as } \xi_{2} \rightarrow \infty .
$$

Taking the inverse transform of (27) and using Eqs. (34) and (38) we have

$$
\begin{aligned}
w\left(0, \xi_{2}\right)= & \frac{1}{2 \pi\left(\delta^{2}+1\right)} \int_{\mathscr{L}}\left\{\left(\delta^{2}-(\eta+1) s^{2}\right) \exp \left[-\xi_{2} \beta(s)\right]\right. \\
& \left.+\left((\eta+1) s^{2}+1\right) \exp \left[-\xi_{2} \alpha(s)\right]\right\} \bar{w}^{-}(s, 0) \mathrm{d} s,
\end{aligned}
$$


and, in consideration of (29), the limit as $\xi_{2} \rightarrow \infty$ is given by

$$
w\left(0, \xi_{2}\right) \rightarrow \frac{1}{2 \pi\left(\delta^{2}+1\right)} \int_{-\delta}^{\delta}\left((\eta+1) s^{2}+1\right) \exp \left[-\xi_{2} \alpha(s)\right] \bar{w}^{-}(s, 0) \mathrm{d} s .
$$

Then, by the method of stationary phase (Bleistein and Handelsman, 1975), it can be shown that $w\left(0, \xi_{2}\right)$ behaves as in Eq. (43). We observe that, setting $\xi_{2}=0$ in Eq. (44), one gets

$$
w(0,0)=\frac{1}{2 \pi\left(\delta^{2}+1\right)} \int_{\mathscr{L}} \bar{w}^{-}(s, 0) \mathrm{d} s=0,
$$

the last equality being obtained by Jordan's lemma.

\section{Full-field solution by the Wiener-Hopf method}

In the present section the Wiener-Hopf analytic continuation technique (Freund, 1990; Noble, 1958; Roos, 1969) is used to obtain the full-field solution for a semi-infinite crack in a half-space subject to a reduced traction shear wave applied to the crack surfaces. For the application of the Wiener-Hopf $(\mathrm{W}-\mathrm{H})$ method, knowledge of the number and of the location of all roots of the kernel function $K(s)$, as defined in Eq. (36), is demanded Noble (1958). Besides, the behaviour of $K(s)$ as $|s| \rightarrow \infty$ is required

$$
K(s)=\mathrm{c}|s|^{3}+O(|s|), \quad \text { as }|s| \rightarrow \infty,
$$

where $c=\frac{1}{2}(\eta+1)(3-\eta)$. With this knowledge, the function $K(s)$ can be factorized into the product of two functions, $K^{ \pm}(s)$, analytic in the corresponding domain $\mathcal{D}^{ \pm}$(Fig. 5). Indeed, let us introduce the function

$$
F(s)=\frac{\alpha(s) K(s)}{c\left(s^{2}-a^{2}\right)\left(s^{2}+b^{2}\right)} \frac{s^{2}-s_{0}^{2}}{s^{2}-s_{3}^{2}},
$$

where $s_{0}$ is a special point defined in Eq. (A.4). The choice of $\beta(s)$ in place of $\alpha(s)$ at the numerator of $F(s)$ is equally possible but it prevents considering the special cases $\eta=0$ and $\delta=0$ within the general framework. Indeed, for $\eta=0$, the roots $\pm a$ and $\pm 1 b$ coincide with the branch points $\pm \delta$ and \pm 1 , respectively, and they become of order $1 / 2$. Similarly, for $\delta=0$, the pair of simple roots $\pm a$ collapse into the simple root $a=0$.

The function $F(s)$ is even, satisfies the reflection principle (37), and it tends to 1 as $|s| \rightarrow \infty$. Besides, it has neither roots nor poles in the cut physical sheet, although it exhibits two branch cuts with the parametrized representation

$$
\mathcal{F}^{ \pm}=\{\mp \delta \pm(\delta+\imath) t, \quad t \in[0,1]\} \subset \mathcal{K}^{ \pm} .
$$

Consequently, it admits the product decomposition $F(s)=F^{+}(s) F^{-}(s)$, where the functions $F^{ \pm}(s)$ are analytic in the domains $\mathcal{D}^{ \pm}$, respectively. Since $F(s)$ is even, we can assume, without loss of generality,

$$
F^{ \pm}(-s)=F^{\mp}(s)
$$

Details of the factorization may be found in Appendix A.

Once the factorization has been accomplished, Eq. (45) provides

$$
K^{ \pm}(s)=\sqrt{c} \frac{(s \mp a)(s \pm \imath b)}{\alpha^{ \pm}(s)} \frac{s \pm s_{3}}{s \pm s_{0}} F^{ \pm}(s),
$$

so that $K^{ \pm}(-s)=K^{\mp}(s)$ and we have the leading term asymptotics

$$
K^{ \pm}(s)=\sqrt{c} \exp ( \pm \imath \pi / 4) s^{3 / 2}, \quad \text { as } \quad|s| \rightarrow \infty,
$$

in the relevant analyticity region. Here, the functions $\alpha(s)$ and $\beta(s)$ are factorized into the product of two functions, named plus and minus, respectively analytic in $\mathcal{D}^{+}$and $\mathcal{D}^{-}$, namely

$$
\alpha^{ \pm}(s)=e^{\mp l \pi / 4} \sqrt{s \mp \delta}, \quad \beta^{ \pm}(s)=e^{\mp l \pi / 4} \sqrt{s \pm l} .
$$

The factor $e^{\mp 1 \pi / 4}$ warrants that we have $\alpha^{ \pm}(-s)=\alpha^{\mp}(s)$ and $\beta^{ \pm}(-s)=\beta^{\mp}(s)$. The W-H Eq. (39) becomes

$$
\frac{p_{3}^{+}(s, 0)}{G K^{+}(s)}=-\frac{1}{2 \lambda^{3}} K^{-}(s) \bar{w}^{-}(s, 0)+\imath \frac{\tau_{0}}{(s+k) K^{+}(s)} .
$$

Therefore, in light of (40), both the l.h.s. and the first term at the r.h.s. in Eq. (49) behave as $O\left(s^{-1}\right)$ as $|s| \rightarrow \infty$, whereas the last term at the r.h.s. behave as $O\left(s^{-5 / 2}\right)$. This last term is a mixed function, yet it may be easily split by inspection into the sum of a plus and a minus function (indeed $-k$ is not a zero for $K^{+}(s)$ )

$$
\frac{p_{3}^{+}(s, 0)}{G K^{+}(s)}-\imath \frac{\tau_{0}}{s+k}\left[\frac{1}{K^{+}(s)}-\frac{1}{K^{+}(-k)}\right]=-\frac{1}{2 \lambda^{3}} K^{-}(s) \bar{w}^{-}(s, 0)+\imath \frac{\tau_{0}}{(s+k) K^{+}(-k)},
$$

so thus the 1.h.s. is a plus function while the r.h.s is a minus function. Therefore, either hand is an entire function in the relevant domain and, in consideration of the common strip of analyticity existing between the two (which is really the line 
$\mathscr{L})$, analytic continuation warrants they are the same entire function $E(s)$. Determination of $E(s)$ hinges on the extended form of Liouville's theorem and on the asymptotic behaviour expected at infinity, see Section 4.1. Consequently, $E(s) \equiv 0$ and

$$
\bar{w}^{-}(s, 0)=2 \imath \frac{\lambda^{3}}{K^{-}(k)} \frac{\tau_{0}}{(s+k) K^{-}(s)},
$$

whose single real pole, in consideration of the definition (48), matches the form (41) and thereby satisfies Sommerfeld's radiation condition.

For the plus transform of the reduced traction we have

$$
\bar{p}_{3}^{+}(s, 0)=\imath G \frac{\tau_{0}}{s+k}\left[1-\frac{K^{+}(s)}{K^{-}(k)}\right],
$$

and the corresponding full-range Fourier transform, evaluated on the crack line, easily follows from Eq. (31)

$$
\bar{p}_{3}(s, 0)=-\imath G \frac{\tau_{0}}{s+k} \frac{K^{+}(s)}{K^{-}(k)} .
$$

\section{Results}

\subsection{Representation of displacement}

The inverse Fourier transform of Eq. (50) gives the displacement along the crack line

$$
w\left(\xi_{1}, 0, \tau\right)=\imath \lambda^{3} \tau_{0} \frac{\exp \imath \Omega \tau}{\pi K^{-}(k)} \int_{\mathscr{L}} \frac{\exp \left(-\imath s \xi_{1}\right)}{(s+k) K^{-}(s)} \mathrm{d} s .
$$

Ahead of the crack tip, displacement can be conveniently determined closing the integration path $\mathscr{L}$ around the top branch cut, $\mathcal{K}^{+}$, and adding the contribution of the poles $s=-k,-a$ and $s=\imath b$, namely

$$
\begin{aligned}
w\left(\xi_{1}, 0, \tau\right)= & -2 \lambda^{3} \tau_{0} \frac{\exp \imath \Omega \tau}{K^{-}(k)}\left[\frac{1}{2 \pi \imath} \int_{\mathcal{K}^{+}} \frac{\exp \left(-\imath s \xi_{1}\right)}{(s+k) K^{-}(s)} \mathrm{d} s+\frac{\exp \left(\imath k \xi_{1}\right)}{K^{+}(k)}\right. \\
& \left.-\frac{\exp \left(\imath a \xi_{1}\right)}{k-a} \frac{\alpha^{+}(a)}{\sqrt{\mathrm{c}}(a+\imath b) F^{+}(a)} \frac{a+s_{0}}{a+s_{3}}+\frac{\exp \left(b \xi_{1}\right)}{k+\imath b} \frac{\alpha^{-}(\imath b)}{\sqrt{\mathrm{c}}(a+\imath b) F^{-}(\imath b)} \frac{\imath b-s_{0}}{\imath b-s_{3}}\right],
\end{aligned}
$$

$$
\xi_{1}<0
$$

We remark that simple poles represent travelling waves. Indeed, the second term in square brackets provides the displacement wave entrained by the traction wave applied at the crack faces, Fig. 6a, while the third term brings outgoing Rayleigh waves, reflected by the crack-tip. The last term is remarkable in that it represents waves that decay exponentially for $\xi_{1}<0$ and yet propagate along the $\xi_{2}$ direction, Fig. $6 c$. To see this, we note that $\alpha(\imath b)=-l \sqrt{b^{2}+\delta^{2}}$ and $\beta(l b)=-l \sqrt{b^{2}-1}$, whence a pair of waves arises with speed

$$
\frac{c_{b 1}}{c_{s}}=\frac{\Omega}{\sqrt{b^{2}+\delta^{2}}} \lambda, \quad \text { and } \quad \frac{c_{b 2}}{c_{s}}=\frac{\Omega}{\sqrt{b^{2}-1}} \lambda .
$$

Such waves may be put to great advantage in non-destructive material testing for they are highly localized along $\xi_{1}$ in correspondence of the crack-tip location. Furthermore, we observe that unlike $c_{b 1}<c_{R} \leq c_{s}, c_{b 2}$ may be very large and greater than the Rayleigh wave speed. The possibility of surface waves moving at super-Rayleigh speed in couple-stress materials has been pointed out in Graff and Pao (1967) and discussed in Ottosen et al. (2000) and Georgiadis and Velgaki (2003), in the context of plane strain. Interestingly, we note that for $\eta=0, s=-a$ and $s=\imath b$ are no longer poles. The first term in square brackets represents non-planar body waves, moving with speed $\tilde{c}$ along the crack line and away from the crack-tip, Fig. 6 b.

It's interesting to observe that unboundedness (resonance) occurs only for $k=-a$, that is when the reduced traction shear wave is associated with Rayleigh Rayleigh waves being fed into the crack-tip. Indeed, when $k=a$ and Rayleigh waves move out of the crack-tip, the second and the third term in square brackets combine into a bounded term.

We now consider the pole $s=-k$ in the context of the full-field solution (44). Eq. (29) shows that the real interval $|k|<\delta$ is associated with a decaying and a propagating wave along $\xi_{2}$, the latter with speed $c \geq c_{R}$ greater than Rayleigh. Indeed, this result corresponds to a super-Rayleigh loading condition. The condition of exponential loading, $k=\imath k_{2},-1<k_{2}<0$, still brings a pair of waves along $\xi_{2}$, one decaying and the other propagating, yet the latter moves with sub-Rayleigh speed $\Omega \lambda c_{s}\left(k_{2}^{2}+\delta^{2}\right)^{-1 / 2}$. However, when decay is strong enough along $\xi_{1}$, i.e. $k_{2}<-1$, the decaying wave turns propagating along $\xi_{2}$ with speed $\Omega \lambda c_{S} / \sqrt{k_{2}^{2}-1}$, which generally exceeds $c_{R}$.

By Jordan's lemma (Roos, 1969, Section 1.5), the displacement $w\left(\xi_{1}, 0\right)$ vanishes beyond the crack tip, in agreement with the boundary condition (19a). The full displacement field beyond the crack-tip is obtained from Eq. (44) and closing the integration path around $\mathcal{K}^{-}$. Then, only body waves moving with speed $\tilde{c}$ along the crack line and away from the crack-tip appear. It is concluded that the crack tip acts as a scatterer of the applied traction shear wave, Fig. $6 \mathrm{~d}$.

Fig. 7 shows the displacement along the crack line for two different values of the traction shear wave wavenumber $k$. 


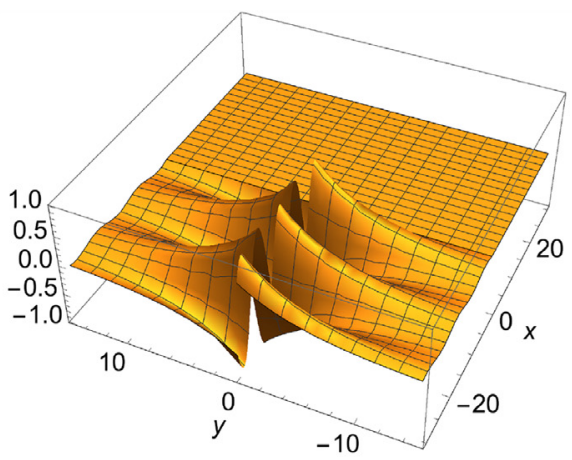

(a) Displacement wave entrained by the traction shear wave applied at the crack faces



(c) Localized wave travelling along $\xi_{2}$

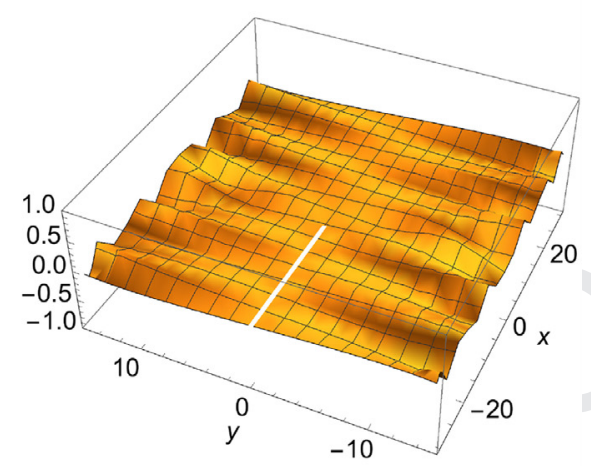

(b) Scattered body wave

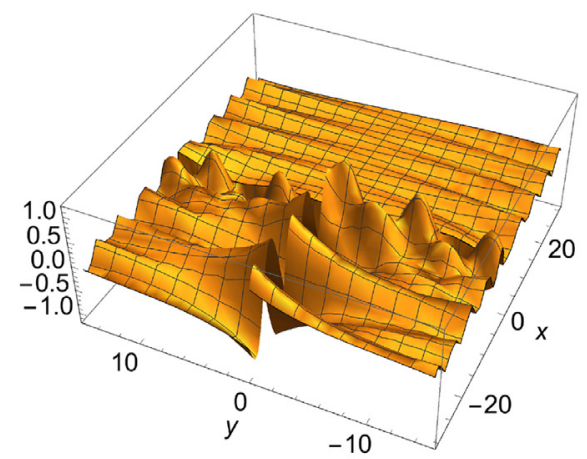

(d) Total wave pattern

Fig. 6. Schematic representation of the wave pattern for $k<-a$ (each wave is scaled as to improve clarity and only one wave is considered for each contribution; reflected Rayleigh waves are disregarded).

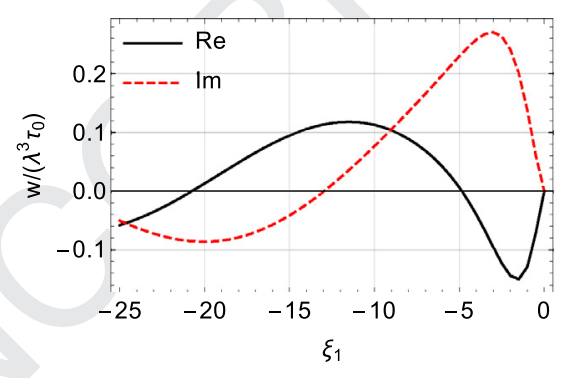

(a) $k=\frac{1}{2}-\imath$

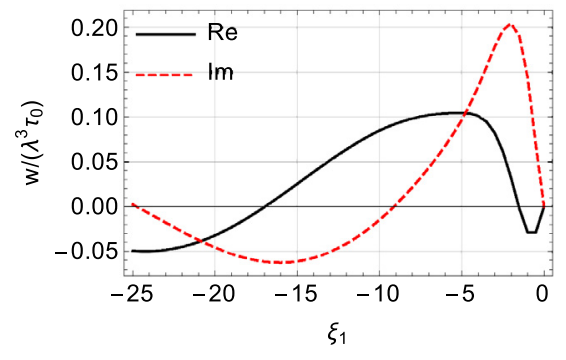

(b) $k=1-\imath$

Fig. 7. Dimensionless displacement $w /\left(\lambda^{3} \tau_{0}\right)$ ahead of the crack tip for $\delta=0.2$ and $\eta=0.797$.

\subsection{Representation of stresses}

The full-range Fourier transform of the symmetric and of the skew-symmetric shear stress along the crack line read

$$
\bar{\sigma}_{23}(s, 0)=-\frac{G}{\lambda\left(1+\delta^{2}\right)}\left\{\left[(1+\eta) s^{2}+1\right] \alpha(s)-\left[(1+\eta) s^{2}-\delta^{2}\right] \beta(s)\right\} \bar{w}^{-}(s, 0),
$$

and

$$
\bar{t}_{23}(s, 0)=-\frac{G}{2 \lambda^{3}\left(1+\delta^{2}\right)}\left\{\left[(1+\eta) s^{2}+1\right] \alpha(s)+\delta^{2}\left[(1+\eta) s^{2}-\delta^{2}\right] \beta(s)\right\} \bar{w}^{-}(s, 0) .
$$

The Fourier transform of the skew-symmetric shear stress, $\bar{\tau}_{23}\left(\xi_{1}, 0\right)$, is then obtained subtracting Eqs. (52) from (53). The Fourier transform of the couple stress is given by

$$
\bar{\mu}_{22}(s, 0)=\imath \lambda^{-1} \ell(1+\eta) s \bar{\sigma}_{23}(s, 0) \text {. }
$$




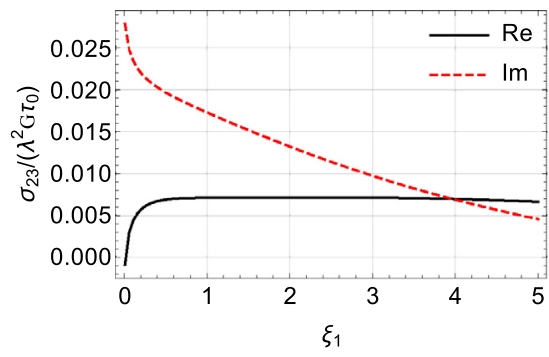

(a) $k=\frac{1}{2}-\imath$

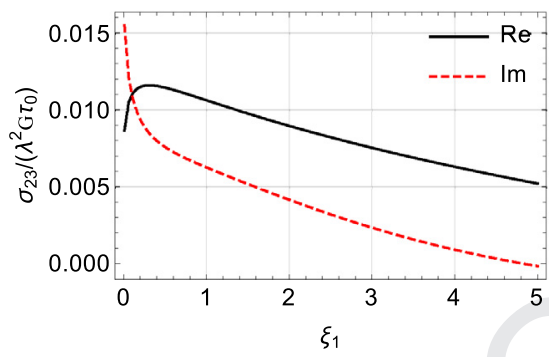

(b) $k=1-\imath$

Fig. 8. Dimensionless symmetric shear stress $\sigma_{23} /\left(\lambda^{2} G \tau_{0}\right)$, beyond the crack-tip, for $\delta=0.2$ and $\eta=-0.7$.

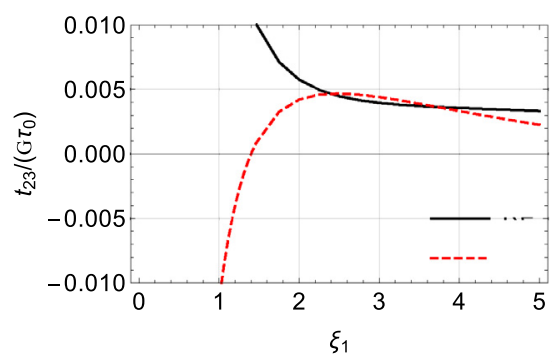

(a) $k=\frac{1}{2}-\imath$

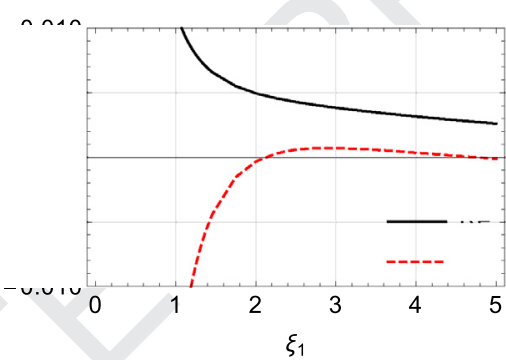

(b) $k=1-\imath$

Fig. 9. Dimensionless total shear stress $t_{23} /\left(G \tau_{0}\right)$, beyond the crack-tip, for $\delta=0.2, \eta=0.797$.

It is important to observe that $\bar{\sigma}_{23}(s, 0), \bar{t}_{23}(s, 0)$ and $\bar{\mu}_{22}(s, 0)$ are not plus functions, although they are analytic in the halfplane $\Im(s)>b$. This analyticity property warrants that the Tauberian theorems may be applied in the asymptotic analysis of Section 6.3. In fact, stresses feature in $\mathcal{D}^{+}$the branch cut $\mathcal{F}^{+}$, coming from $F^{-}(s)$, alongside the simple poles $s=-a, 1 b$ and $s=-k$, originating from $\bar{w}^{-}(s, 0)$. Such poles provide travelling wave contributions to the stress field ahead of the crack tip, as described for displacement.

Beyond the crack tip, integration in the inverse Fourier transform for stresses is conveniently carried out closing the integration path $\mathscr{L}$ around the lower branch cut $\mathcal{K}^{-}$. In particular, such deformation of the integration path is necessary to perform the inverse Fourier transform of Eq. (53), in consideration of the divergent asymptotic behaviour of $\bar{t}_{23}$ as $|s| \rightarrow+\infty$, see Eq. (56). Like for displacement, there are no travelling waves contributing to stress beyond the crack-tip. Indeed, we have the representation formulas for body stress waves

$$
\sigma_{23}\left(\xi_{1}, 0, \tau\right)=2 \lambda^{2} G \tau_{0} \frac{\sqrt{1-\imath \delta}}{\pi(\delta-\imath) K^{-}(k)}\left[I_{0}^{(1)}\left(\xi_{1}\right)-I_{1}^{(1)}\left(\xi_{1}\right)\right] \exp \left[\imath\left(-\delta \xi_{1}+\Omega \tau\right)\right],
$$

$$
t_{23}\left(\xi_{1}, 0, \tau\right)=G \tau_{0} \frac{\sqrt{1-\imath \delta}}{\pi(\delta-\imath) K^{-}(k)}\left[I_{0}^{(1)}\left(\xi_{1}\right)+\delta^{2} I_{1}^{(1)}\left(\xi_{1}\right)\right] \exp \left[\imath\left(-\delta \xi_{1}+\Omega \tau\right)\right],
$$

where $n \in\{1,2\}$,

$$
I_{0,1}^{(n)}\left(\xi_{1}\right)=\int_{0,1}^{\infty} f_{0,1}^{(n)}(t) \exp \left[(1 \delta-1) t \xi_{1}\right] \mathrm{d} t,
$$

and

$$
\begin{aligned}
f_{0}^{(n)}(t)=\frac{\left[(1+\eta) s(t)^{2}+1\right]^{n} \alpha^{-}(s(t))}{[s(t)+k] K^{-}(s(t))} \sqrt{t}, & t>0, \\
f_{1}^{(n)}(t)=\frac{\left[(1+\eta) s(t)^{2}-\delta^{2}\right]^{n} \beta^{-}(s(t))}{[s(t)+k] K^{-}(s(t))} \sqrt{t-1}, & t>1 .
\end{aligned}
$$

The behaviour of the symmetric and of the total stress is shown in Figs. 8 and 9, while couple stress is plotted in Fig. 10.

From Eq. (51), we write the inversion formula for the reduced total traction

$$
p_{3}\left(\xi_{1}, 0, \tau\right)=-\imath G \tau_{0} \frac{\exp \imath \Omega \tau}{2 \pi} \int_{\mathscr{L}} \frac{\exp \left(-\imath s \xi_{1}\right)}{s+k} \frac{K^{+}(s)}{K^{-}(k)} \mathrm{d} s,
$$






(a) $k=\frac{1}{2}-\imath$

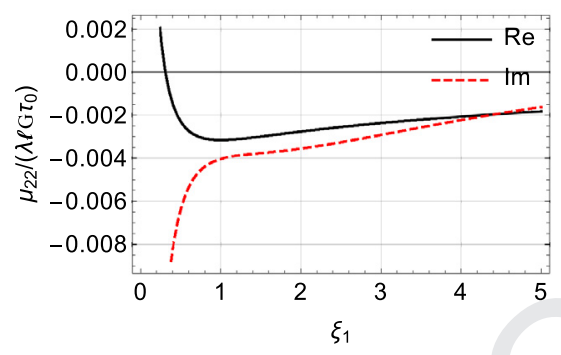

(b) $k=1-\imath$

Fig. 10. Dimensionless couple stress $\mu_{22} /\left(\lambda \ell G \tau_{0}\right)$, beyond the crack-tip, for $\delta=0.2$ and $\eta=0.797$.

that, ahead of the crack-tip, reduces to the contribution of the pole $s=-k$ reproducing the applied shear wave. Beyond the crack-tip, we write $K^{+}(s)=K(s) / K^{-}(s)$ and

$$
\bar{p}_{3}(s, 0)=-G \frac{K(s)}{2 \lambda^{3}} \bar{w}^{-}(s, 0) .
$$

Upon deforming the integration path around $\mathcal{K}^{-}$, we get the representation formula for reduced traction body waves

$$
p_{3}\left(\xi_{1}, 0, \tau\right)=G \tau_{0} \frac{\exp \left[\imath\left(-\delta \xi_{1}+\Omega \tau\right)\right]}{2 \pi(1+\imath \delta) K^{-}(k)}\left[I_{0}^{(2)}\left(\xi_{1}\right)-I_{1}^{(2)}\left(\xi_{1}\right)\right] .
$$

\subsection{Dynamic stress intensity factors}

Eq. (50) immediately provides us with the asymptotics

$$
\bar{w}^{-}(s, 0)=2 \imath^{3 / 2} \lambda^{3} \tau_{0} \frac{1}{\sqrt{\mathrm{c}} K^{-}(k)} s^{-5 / 2}, \quad \text { as } \quad|s| \rightarrow \infty,
$$

valid in the analyticity region $\mathcal{D}^{-}$(we neglect the harmonic term throughout this Section). Then, the Tauberian theorem (Roos, 1969, Section 2.14) lends the following asymptotic representation for the inverse Fourier transform

$$
\ell w\left(x_{1}, 0\right)=\text { r.b.m. }+K_{\mathrm{III}}^{w}\left(-x_{1}\right)^{3 / 2}, \quad \text { as } \quad x_{1} \rightarrow 0^{-}
$$

where r.b.m. is an inessential rigid body motion and

$$
K_{\mathrm{III}}^{w}=-16 \lambda^{3 / 2} \tau_{0} \frac{1}{3 \sqrt{2 \pi \ell(1+\eta)(3-\eta)} K^{-}(k)} .
$$

Asymptotics for the symmetric, total and couple stress, respectively Eqs. (52), (53) and (54), are similarly obtained

$$
\begin{aligned}
\bar{\sigma}_{23}(s, 0) & =-l^{3 / 2} \lambda^{2} G \tau_{0} \frac{1-\eta}{\sqrt{\mathrm{c}} K^{-}(k)} s^{-3 / 2}, \\
\bar{t}_{23}(s, 0) & =-l^{3 / 2} G \tau_{0} \frac{1+\eta}{\sqrt{\mathrm{c}} K^{-}(k)} \sqrt{s}, \quad \text { as }|s| \rightarrow \infty . \\
\bar{\mu}_{22}(s, 0) & =l^{1 / 2} \lambda \ell G \tau_{0} \frac{1-\eta^{2}}{\sqrt{\mathrm{c}} K^{-}(k)} s^{-1 / 2},
\end{aligned}
$$

Then, for the respective inverse Fourier transforms, we infer the asymptotic behaviour

$$
\begin{aligned}
\sigma_{23}\left(x_{1}, 0\right) & =\Sigma_{23}+K_{\mathrm{III}}^{\sigma} \sqrt{x_{1}}, \\
t_{23}\left(x_{1}, 0\right) & =K_{\mathrm{III}}^{\mathrm{t}} x_{1}^{-3 / 2}, \quad \text { as } x_{1} \rightarrow 0^{+}, \\
\mu_{22}\left(x_{1}, 0\right) & =K_{\mathrm{III}}^{\mu} x_{1}^{-1 / 2},
\end{aligned}
$$

where $\Sigma_{23}$ can be determined evaluating Eq. (55a) at $\xi_{1}=0$. Here, we have let the dynamic stress intensity factors for stresses

$$
\begin{aligned}
& K_{\mathrm{III}}^{\sigma}=-4 \lambda^{3 / 2} G \tau_{0} \frac{1-\eta}{\sqrt{2 \pi \ell(3-\eta)(1+\eta)} K^{-}(k)}, \\
& K_{\mathrm{III}}^{t}=-(\lambda \ell)^{3 / 2} G \tau_{0} \sqrt{\frac{1+\eta}{2 \pi(3-\eta)}} \frac{1}{K^{-}(k)}, \\
& K_{\mathrm{III}}^{\mu}=-2(1-\eta) K_{\mathrm{III}}^{t} .
\end{aligned}
$$


Such expressions generalize to the dynamic regime the asymptotic results (Radi, 2008, Eqs. (38)) obtained for static application of the far-field loading $K_{\mathrm{III}}$, valid in linear elastic fracture mechanics (LEFM). Indeed, the correction accounts for the important role of frequency of the applied loading on stress concentration (Graff and Pao, 1967). Formal correspondence with LEFM results is met by taking

$$
K_{\mathrm{III}}=2 G \tau_{0} \sqrt{\lambda^{3} \ell} \frac{1}{K^{-}(k)} .
$$

This expression may be explained looking at the far-field behaviour of $w(r, \vartheta=0), r \rightarrow+\infty$, which is obtained by investigating the behaviour of $\bar{w}(s, 0)$ as $s \rightarrow 0$. In the general case, $\bar{w}^{-}(s, 0)$ behaves like a constant as $s \rightarrow 0$ and thereby $w(r)$ decays as $r^{2}$. In the static limit we have $\delta=0, \lambda=2^{-1 / 2}$ and, for $k \ll s$, we get $K^{-}(s) \sim \sqrt{K(s)} \sim-\sqrt{\imath s}$ and

$$
\bar{w}^{-}(s, 0)=2 \imath^{3 / 2} \frac{\lambda^{3}}{\sqrt{k}} \frac{\tau_{0}}{s^{3 / 2}},
$$

whence, by the Tauberian theorem,

$$
\ell w\left(x_{1}, 0\right)=\frac{2 K_{\mathrm{III}}}{G} \sqrt{\frac{x_{1}}{2 \pi}},
$$

which is the far-field behaviour of LEFM (Zhang et al., 1998).

\section{Conclusions}

Diffraction of reduced traction shear waves applied at the faces of a semi-infinite rectilinear crack in an elastic halfspace with microstructure is considered. Microstructure is accounted for through the indeterminate theory of couple stress elasticity and motion is restricted to antiplane deformation. The full-field solution is obtained in closed form through the Wiener-Hopf technique and it may be adopted as a building block for the solution of general wave scattering problems in a cracked couple-stress half-space. A rather involved wave pattern appears, especially when compared to the simple scenario of classical elasticity. Indeed we find

1. entrained waves moving along the crack line with the applied loading speed, which may be either decaying away from the crack, in the sub-critical regime, or propagating, in the super-critical regime. Transition from a sub-critical to a super-critical regime occurs at the Rayleigh speed, which is wavenumber dependent (dispersion);

2. a pair of Rayleigh waves confined to the crack faces and reflected from the crack-tip;

3. a pair of waves highly localized near the crack-tip and moving away from it, which may be either decaying and propagating with sub-Rayleigh speed or propagating at sub-Rayleigh and super-Rayleigh speed, respectively;

4. body waves scattered by the crack-tip.

Special situations are connected to

- a loading moving toward the crack-tip at Rayleigh speed, because resonance occurs, i.e. solution is unbounded;

- an exponentially-decaying loading with harmonic time variation, for the associated phase speed is infinite;

- $\eta=0$, because waves 2 and 3 disappear;

- $h_{0}=h_{0 c r}=2^{-1 / 2}$ and $\eta=0$, for then the classical solution is retrieved, namely Rayleigh waves collapse into classical non-dispersive shear waves;

- the static regime $\Omega=0$, for which Rayleigh waves collapse into classical shear waves.

Finally, dynamic stress intensity factors are determined for the symmetric stress, the couple-stress, the total stress and the reduced traction. They generalize to the dynamic regime the corresponding expression already obtained for the static application of the classical elastic Mode III solution in the far-field. The correction term brings out the important role of the loading frequency on the stress intensity factors.

\section{Declaration of interest}

Authors have no competing interest to declare.

\section{Acknowledgements}

The authors wish to thank Prof. G. Mishuris for his valuable comments. AV acknowledges the ERC Grant "Instabilities" for supporting his short stay in Trento University working on this paper. AN gratefully acknowledges support from National Group of Mathematical Physics (GNFM-INdAM) through the Young researchers fellowship programme 2017, Prot. n. U UFMBAZ-2017/0000259 08/06/2017. 


\section{Appendix A. Factorization of $F(s)$}

In the logarithmic factorization, Cauchy theorem is exploited to split $\ln F(s)$ into the sum of two functions analytic in the regions $\mathcal{D}^{ \pm}$, respectively,

$$
\ln F^{ \pm}(s)=\frac{1}{2 \pi \imath} \int_{\mathscr{L}} \frac{\ln F(\zeta)}{\zeta-s} \mathrm{~d} \zeta
$$

Due to the analytical property of $F(s)$ and Jordan's lemma, the integration path $\mathscr{L}$ can be closed around the upper branch cut $\mathcal{F}^{+}$. The contributions from the branch points is vanishingly small. The upper branch cut is parametrized as in Eq. (46). In the special case $\delta=0$, the branch cut corresponds to the interval on the imaginary axis $\{1 t, t \in[0,1]\}$.

The ratio $\alpha(s) / \beta(s)$ appearing in the function $F(s)$ jumps across the branch cut according to

$$
\lim _{\epsilon \rightarrow 0^{+}} \frac{\alpha(\zeta(t) \pm \imath \epsilon)}{\beta(\zeta(t) \pm \imath \epsilon)}= \pm \imath \sqrt{\frac{t}{1-t} \frac{(2-t) \delta-\imath t}{(1-t) \delta-\imath(1+t)}}, \quad t \in[0,1]
$$

Eq. (46) yields

$$
\ln F^{-}(s)=\frac{\delta+\imath}{2 \pi \imath} \int_{0}^{1} \lim _{\epsilon \rightarrow 0}[\ln F(\zeta(t)-\imath \epsilon)-\ln F(\zeta(t)+\imath \epsilon)] \frac{\mathrm{d} t}{\zeta(t)-s},
$$

and using

$$
\begin{aligned}
\ln F(\zeta(t)-\imath \epsilon)-\ln F(\zeta(t)+\imath \epsilon) & =\ln \frac{F(\zeta(t)-\imath \epsilon)}{F(\zeta(t)+\imath \epsilon)} \rightarrow \\
& =\ln \frac{1-\imath \psi(t)}{1+\imath \psi(t)}=-2 \imath \arctan \psi(t)=-2 \imath\left(\pi / 2-\arctan \psi^{-1}(t)\right),
\end{aligned}
$$

with

$$
\psi(t)=\left(\frac{(1+\eta)[(\delta+\imath) t-\delta]^{2}-\delta^{2}}{(1+\eta)[(\delta+\imath) t-\delta]^{2}+1}\right)^{2} \sqrt{\frac{(1-t)^{2} \delta-\imath\left(1-t^{2}\right)}{(2-t) \delta t-\imath t^{2}}}
$$

we obtain

$$
\ln F^{-}(s)=\frac{\delta+\imath}{\pi} \int_{0}^{1} \frac{\arctan \psi(t)}{\delta-(\delta+\imath) t+s} \mathrm{~d} t=G^{-}(s) .
$$

In the static case $\delta=0$, Eq. (A.2) gives the corresponding function introduced in (Radi, 2008, Eq. (59))

$$
\psi(t)=\frac{t^{3} \sqrt{1-t^{2}}}{\left(t^{2}-\frac{1}{\eta+1}\right)^{2}}
$$

In this situation, a pole of $\psi(t)$ is encountered at $t=1 / \sqrt{1+\eta}$, inasmuch as $\eta>0$, although this brings no harm to the evaluation of $G^{-}(s)$, in light of the arctangent being bounded. In contrast, special care must be paid in the evaluation of the contour integral when the root $s_{3}$ lays inside the branch cut, as this may bring a $2 \pi 1$ jump when transforming the difference of the logarithms in Eq. (A.1). Clearly, from Eq. (A.3) and the property (47), we have

$$
F^{\mp}(s)=\exp G^{\mp}(s)
$$

where $G^{ \pm}(-s)=G^{\mp}(s)$. Observing that the factorization is independent of the location of $s_{0}$ and enforcing Eq. (45) to hold at $s=0$ brings the requirement

$$
s_{0}=\imath a b s_{3} \sqrt{\mathrm{c} \frac{F^{+}(0) F^{-}(0)}{\alpha(0) K(0)}} .
$$

In particular, the point $s_{0}$ coincides with $s_{3}$ when the function $K(s)$ admits two pairs of roots in the physical sheet, while it is conveniently located in the branch cut when $K(s)$ admits three root pairs.

\section{Supplementary material}

Supplementary material associated with this article can be found, in the online version, at doi:10.1016/j.jmps.2018.11.013.

\section{References}

Achenbach, J., 1984. Wave propagation in elastic solids. Applied Mathematics and Mechanics, 16. Elsevier, North-Holland.

Bleistein, N., Handelsman, R.A., 1975. Asymptotic Expansions of Integrals. Courier Corporation.

Clebsch, A., 1863. Ueber die reflexion an einer kugelfläche. J. Reine Angew. Math. 61, 195-262.

Destrade, M., Fu, Y., Nobili, A., 2016. Edge wrinkling in elastically supported pre-stressed incompressible isotropic plates. Proc. R. Soc. A 472 (2193), 20160410. 
Eremeyev, V.A., Rosi, G., Naili, S., 2016. Surface/interfacial anti-plane waves in solids with surface energy. Mech. Res. Commun. 74, 8-13.

Eremeyev, V.A., Rosi, G., Naili, S., 2018. Comparison of anti-plane surface waves in strain-gradient materials and materials with surface stresses. Math. Mech. Solids. doi: 1081286518769960.

Eringen, A.C., 1999. Theory of micropolar elasticity. In: Microcontinuum Field Theories. Springer, pp. 101-248.

Freund, L.B., 1990. Dynamic fracture mechanics. Cambridge Monographs on Mechanics and Applied Mathematics. Cambridge university press, Cambridge.

Gao, X.L., Ma, H.M., 2010. Solution of Eshelby's inclusion problem with a bounded domain and Eshelby's tensor for a spherical inclusion in a finite spherical matrix based on a simplified strain gradient elasticity theory. J. Mech. Phys. Solids 58 (5), 779-797.

Georgiadis, H.G., 2003. The mode III crack problem in microstructured solids governed by dipolar gradient elasticity: static and dynamic analysis. J. Appl. Mech. 70 (4), 517-530.

Georgiadis, H.G., Vardoulakis, I., 1998. Anti-plane shear Lamb's problem treated by gradient elasticity with surface energy. Wave Motion 28 (4), $353-366$.

Georgiadis, H.G., Velgaki, E.G., 2003. High-frequency Rayleigh waves in materials with micro-structure and couple-stress effects. Int. J. Solids Struct. 40 (10), $2501-2520$

Gourgiotis, P.A., Georgiadis, H.G., Neocleous, I., 2013. On the reflection of waves in half-spaces of microstructured materials governed by dipolar gradient elasticity. Wave Motion 50 (3), 437-455.

Graff, K.F., Pao, Y.H., 1967. The effects of couple-stresses on the propagation and reflection of plane waves in an elastic half-space. J. Sound Vib. 6 (2), 217-229.

Harris, J.G., 2001. Linear elastic waves. Cambridge Texts in Applied Mathematics, 26. Cambridge University Press.

Kaplunov, J., Nobili, A., 2017. A robust approach for analysing dispersion of elastic waves in an orthotropic cylindrical shell. J. Sound Vib. 401, 23-35.

Koiter, W.T., 1964. Couple-stress in the theory of elasticity. In: Proc. K. Ned. Akad. Wet, 67. North Holland Pub, pp. 17-44.

Lakes, R.S., 1986. Experimental microelasticity of two porous solids. Int. J. Solids Struct. 22 (1), 55-63.

Lam, D.C.C., Yang, F., Chong, A., Wang, J., Tong, P., 2003. Experiments and theory in strain gradient elasticity. J. Mech. Phys. Solids 51 (8), 1477-1508.

Maranganti, R., Sharma, P., 2007. A novel atomistic approach to determine strain-gradient elasticity constants: tabulation and comparison for various metals, semiconductors, silica, polymers and the (ir) relevance for nanotechnologies. J. Mech. Phys. Solids 55 (9), $1823-1852$.

Mindlin, R.D., 1964. Micro-structure in linear elasticity. Arch. Ration. Mech. Anal. 16 (1), 51-78.

Mindlin, R.D., Eshel, N.N., 1968. On first strain-gradient theories in linear elasticity. Int. J. Solids Struct. 4 (1), $109-124$.

Mishuris, G., Piccolroaz, A., Radi, E., 2012. Steady-state propagation of a mode III crack in couple stress elastic materials. Int. J. Eng. Sci. 61, 112-128.

Morini, L., Piccolroaz, A., Mishuris, G., 2014. Remarks on the energy release rate for an antiplane moving crack in couple stress elasticity. Int. J. Solids Struct. 51 (18), 3087-3100.

Morini, L., Piccolroaz, A., Mishuris, G., Radi, E., 2013. On fracture criteria for dynamic crack propagation in elastic materials with couple stresses. Int. J. Eng. Sci. 71, 45-61.

Mow, C.C., Pao, Y.H., 1971. The Diffraction of Elastic Waves and Dynamic Stress Concentrations. Technical report. RAND Corporation, Santa Monica, California. Nakamura, S., Lakes, R.S., 1995. Finite element analysis of saint-venant end effects in micropolar elastic solids. Eng. Comput. 12 (6), $571-587$.

Nobili, A., Radi, E., Lanzoni, L., 2017. Flexural edge waves generated by steady-state propagation of a loaded rectilinear crack in an elastically supported thin plate. In: Proc. R. Soc. A, 473. The Royal Society, p. 20170265.

Noble, B., 1958. Methods Based on the Wiener-Hopf Technique for the Solution of Partial Differential Equations. International Series of Monographs on Pure and Applied Mathematics, 7. Pergamon Press, New York.

Ottosen, N.S., Ristinmaa, M., Ljung, C., 2000. Rayleigh waves obtained by the indeterminate couple-stress theory. Eur. J. Mech.-A/Solids 19 (6), $929-947$.

Radi, E., 2008. On the effects of characteristic lengths in bending and torsion on mode iii crack in couple stress elasticity. Int. J. Solids Struct. 45 (10), 3033-3058.

Roos, B.W., 1969. Analytic Functions and Distributions in Physics and Engineering. John Wiley and Sons, Inc., New York, USA.

Shodja, H.M., Goodarzi, A., Delfani, M.R., Haftbaradaran, H., 2015. Scattering of an anti-plane shear wave by an embedded cylindrical micro-/nano-fiber within couple stress theory with micro inertia. Int. J. Solids Struct. 58, 73-90.

Strutt, J.W., 1877. The Theory of Sound, 1. Macmillan and Co.

Yang, F., Chong, A.C.M., Lam, D.C.C., Tong, P., 2002. Couple stress based strain gradient theory for elasticity. Int. J. Solids Struct. 39 (10), $2731-2743$.

Zhang, L., Huang, Y., Chen, J.Y., Hwang, K.C., 1998. The mode III full-field solution in elastic materials with strain gradient effects. Int. J. Fract. 92 (4), $325-348$. 